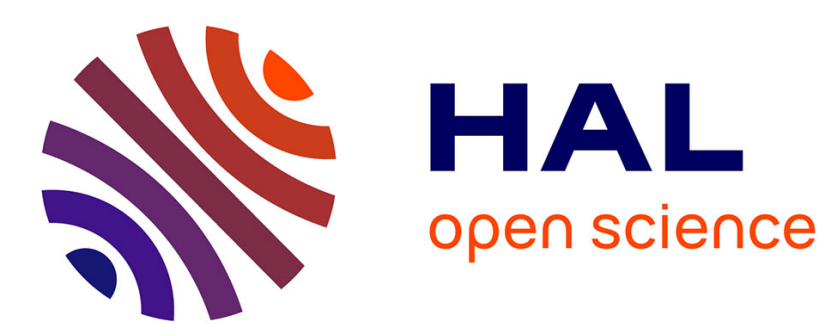

\title{
Assumer les incertitudes dans un marché en transition, un sensemaking prudent
}

Thomas Reverdy

\section{To cite this version:}

Thomas Reverdy. Assumer les incertitudes dans un marché en transition, un sensemaking prudent. Revue Française de Gestion, 2010, 203, pp.101-118. halshs-00487201

\section{HAL Id: halshs-00487201 https://shs.hal.science/halshs-00487201}

Submitted on 28 May 2010

HAL is a multi-disciplinary open access archive for the deposit and dissemination of scientific research documents, whether they are published or not. The documents may come from teaching and research institutions in France or abroad, or from public or private research centers.
L'archive ouverte pluridisciplinaire HAL, est destinée au dépôt et à la diffusion de documents scientifiques de niveau recherche, publiés ou non, émanant des établissements d'enseignement et de recherche français ou étrangers, des laboratoires publics ou privés. 


\section{Assumer les incertitudes dans un marché en transition : un sensemaking prudent}

Thomas Reverdy

PACTE, Université de Grenoble

Adresse PACTE UPMF (le Patio), 38040 Grenoble Cedex 9, France, tél : +33 (0)4 768255 28, e-mail : thomas.reverdy@upmf-grenoble.fr

Résumé

Cet article prend appui sur l'expérience de la libéralisation du marché gazier en Europe continentale pour interroger l'action en situation d'incertitude. Notre enquête auprès de fournisseurs et auprès des acheteurs industriels de gaz dévoile les activités intersubjectives de construction de sens qui contribuent à l'institutionnalisation progressive de représentations et de pratiques. Nous mettons en valeur le paradoxe suivant : pour accroître leur légitimité les professionnels du marché défendent un sensemaking «offensif », avec des stratégies de plus en plus formalisées et sophistiquées; les remises en question les conduit à adapter leur attitude vers un sensemaking plus «prudent» qui se montre plus robuste face aux événements.

Abstract

This article leans on the experience of gas market liberalization in continental Europe and questions the action in uncertainty. Interviews of gas suppliers and purchasers reveal intersubjective sensemaking that contributes to the institutionalization of shared meanings and practices. The article highlights the following paradox : in order to increase their legitimacy, traders defend an offensive sensemaking with formalized and sophisticated practices ; but 
many events challenge their practices and incite them to adapt their attitude for a more prudent sensemaking, more robust against unexpected events.

Mots clefs: Libéralisation, Financiarisation, Marché gazier, Sensemaking, Travail d'institutionnalisation

Présentation de l'auteur

Thomas Reverdy est Maître de Conférences en Sociologie Industrielle. Il enseigne la théorie des organisations à l'INP de Grenoble (Génie Industriel) et participe au laboratoire PACTE. Ses recherches portent sur la façon dont des changements institutionnels se traduisent dans les pratiques et dans les organisations: pressions sociétales et environnementales, risques technologiques, mondialisation des échanges, libéralisation des marchés... 


\title{
Assumer les incertitudes dans un marché en transition : une construction \\ de sens prudente
}

\author{
Thomas Reverdy
}

La politique européenne de libéralisation du secteur énergétique s'est traduite par une ouverture du marché gazier pour les grands consommateurs industriels français à partir de l'année 2000. Pour tirer au mieux parti de l'ouverture des marchés et définir leur stratégie d'achat, ces clients industriels ont cherché à mieux comprendre la logique de formation du prix du gaz et donc les fondamentaux du marché.

Or, dans toute cette période, les fondamentaux du marché (en particulier les pratiques d'approvisionnement des principaux fournisseurs auprès des pays producteurs) sont restés confus et incertains, tant pour des raisons économiques qu'institutionnelles. Il était annoncé qu'avec le développement de la concurrence, les contrats d'approvisionnement à long terme laisseraient la place à un vaste marché spot du gaz, transparent et ouvert, offrant une référence de prix commune à tous les acteurs. Or le marché spot du gaz existe, sans pour autant s’imposer : les contrats à long terme avec les pays producteurs (Norvège, Russie, Algérie...) restent dominants. Plusieurs références de prix coexistent : un prix du gaz indexé sur les produits pétroliers (contrats à long terme) et un prix du gaz fixé par le marché spot.

Pour les grands clients industriels, l'incertitude ne porte pas uniquement sur la variabilité d'un prix du gaz dans un marché où les règles de fonctionnement et le périmètre seraient définis. Elle porte sur l'organisation même de ce marché, sur ses fondamentaux, sur ses règles de fonctionnement, sur son périmètre, et sur les possibilités d'anticiper les mouvements des prix.

Comme le marché du gaz n'est pas stabilisé, les pratiques d'achat de gaz ne sont pas stabilisées non plus. Consultants, fournisseurs et traders prescrivent chacun des «bonnes 
pratiques » différentes : appel d'offre, couverture des risques, suivi des variations de prix. Ils véhiculent aussi des représentations et des interprétations cohérentes avec les pratiques qu'ils défendent. La tentation est grande de gommer la complexité et les incertitudes du marché, d'insister sur certaines caractéristiques, et d'argumenter pour des solutions sophistiquées que chacun maîtrise. Néanmoins, comme le fonctionnement du marché lui-même n'est pas stabilisé, des signaux contradictoires (des fortes variations de prix) peuvent réintroduire le doute quant à la fiabilité des représentations simplifiées, voire les invalider, et invalider les stratégies et les pratiques associées.

Le marché gazier continental se présente donc comme un champ institutionnel émergeant (Maguire et al., 2004) où les connaissances, les règles et les pratiques ne sont pas stabilisées, où des acteurs sont en compétition pour institutionnaliser des pratiques qui leur sont favorables. Il nous offre l'opportunité d'étudier comment des acteurs d'un champ émergeant constituent leur légitimité auprès de leurs interlocuteurs.

L'institutionnalisation de nouvelles pratiques et de nouvelles représentations tient à des dynamiques microsociologiques d'interprétation et de construction de sens, de « sensemaking »(Weber et Glynn, 2006). Les activités de construction de sens visent à réduire ces dissonances cognitives introduites par des signaux contradictoires (Weick, 1993). Ces activités de sensemaking sont des opérations cognitives individuelles mais peuvent aussi être intersubjectives : les individus mettent en commun leurs interprétations et s'influencent mutuellement. Certaines personnes, du fait de leur expérience, de leur compétence, de leur position, ont une influence sur l'activité de sensemaking d'autres personnes. Enfin, il y a institutionnalisation quand les dissonances cognitives sont définitivement surmontées et que les interprétations et les pratiques sont stabilisées, communément partagées, tenues pour évidentes. 
Cette étude de cas est l'occasion de mettre en valeur le paradoxe suivant : afin d'asseoir leur légitimité, les acteurs présents dans un champ institutionnel émergeant ont besoin de rationaliser leurs pratiques, de défendre des compétences spécialisées, une technicité. Ils ont aussi besoin de communiquer une représentation du contexte dans laquelle la pratique qu'ils défendent est pertinente. Il y a institutionnalisation si la représentation du contexte est partagée par tous et devient une évidence, au quel cas les pratiques associées sont tenues pour rationnelles et efficaces. Or il n'y a pas de raison pour que ce processus d'institutionnalisation soit univoque. Plusieurs modèles d'action et plusieurs représentations du contexte peuvent être en compétition. Dans ce cas, le processus d'institutionnalisation est contrarié : aucune pratique et représentation ne parvient à être tenue pour évidente.

Nous proposons donc de distinguer un «sensemaking défensif », où les individus surmontent les dissonances cognitives en conservant les repères cognitifs déjà institutionnalisés, un « sensemaking offensif », où ils abandonnent complètement les repères institutionnalisés pour en adopter de nouveaux au risque d'une simplification abusive de la complexité et de l'incertitude du réel et un «sensemaking prudent » acceptant une pluralité de représentations et de pratiques.

Nous proposons d'explorer l'hypothèse suivante : dans un contexte marqué par une forte incertitude, un «sensemaking défensif » reproduit les institutions existantes et freine l'institutionnalisation de nouvelles pratiques, alors qu'un « sensemaking offensif » contribue à une institutionnalisation rapide de nouvelles pratiques, mais cette institutionnalisation reste fragile face aux incertitudes du contexte, et un «sensemaking prudent » contribue à une institutionnalisation «partielle» mais cette institutionnalisation a plus de chance d'être « robuste » face aux aléas. 
Notre article porte sur l'approvisionnement en gaz dans les pays d'Europe continentale et décrit les stratégies des acteurs de ce marché, comment ils rivalisent pour définir les bonnes pratiques d'achat. En analysant les pratiques d'achat des clients industriels et leur évolution, nous montrerons que l'accompagnement commercial joue un rôle clef dans l'adoption des pratiques d'achat, et qu'il influence la «construction de sens » par les acheteurs. La manière dont il réinterprète au fur et à mesure les événements, permet à l'acheteur d'actualiser sa stratégie et de sophistiquer ses pratiques, malgré un environnement économique qui reste insaisissable. Nous essayons de qualifier, selon les cas, les activités de sensemaking comme défensives, offensives ou prudentes. L'article conclut sur la contribution de l'activité intersubjective de construction de sens à la légitimation et l'institutionnalisation de nouvelles pratiques.

\section{Méthodologie}

Cet article puise dans une enquête menée auprès de grands clients industriels, de fournisseurs et de banques entre 2004 à 2007. Elle est fondée principalement sur des entretiens semidirectifs approfondis auprès d'acheteurs, de responsables opérationnels, de commerciaux vendeurs de gaz ou de produits financiers (40 entretiens d'environ 2 heures, pour 12 clients industriels, une banque et un fournisseur ${ }^{1}$ ). Pour ces clients industriels, le gaz représente entre 5 et $30 \%$ de l'ensemble de leurs coûts d'achat. Ces industriels avaient une expérience de plusieurs années du marché libéralisé. Le croisement des récits nous a permis de reconstituer l'historique des pratiques d'achat de chaque client, de repérer les changements et identifier leurs causes, en particulier la place que tiennent les événements extérieurs concernant le marché gazier mais aussi l'intervention de consultants ou l'apport de conseils et d'information par les commerciaux de divers fournisseurs et banques. Dans une approche essentiellement narrative et inductive (Langley, 1999) nous avons dressé un historique pour chaque client. Ces historiques ont fait l'objet d'une analyse transversale et comparative. Pour chaque client, et chaque étape clef, le croisement des entretiens des acheteurs et commerciaux nous ont permis de reconstituer les activités intersubjectives de construction de signification : comment une argumentation pouvait ou non convaincre un interlocuteur.

\footnotetext{
${ }^{1}$ Pour des raisons de confidentialité nous nous sommes engagés à ne citer aucun nom de fournisseur, ni de banque, ni de consultant, ni de client. Les interviews auprès des clients, l'analyse des offres des fournisseurs, montrent que les stratégies des fournisseurs dominants en Europe continentale sont assez similaires d'un pays à l'autre.
} 
Lors de cette enquête, nous avons été confrontés à de nombreuses reprises à des situations où des événements extérieurs (une évolution soudaine des prix par exemple) pouvaient invalider les croyances forgées antérieurement, discréditer certains discours, certaines pratiques, fragiliser la confiance dans les interlocuteurs privilégiés. Nous avons aussi rencontré des situations où il y avait contradiction entre la stratégie formalisée antérieurement et un nouveau contexte d'action inattendu. L'évocation par les acheteurs, des situations d'inconfort et de doutes, a permis de révéler des situations où les acheteurs hésitaient entre plusieurs références différentes, considérées comme légitimes mais vécues comme contradictoires.

\section{Pluralite des modeles de marches gaziers}

Il existe deux types de marché gazier en Europe, selon que les pays sont producteurs de gaz en grande quantité ou importateurs de gaz. La Grande-Bretagne possède une activité de production de gaz sur son sol : le gaz est vendu par les producteurs aux différents fournisseurs sur des «places de marché » ${ }^{2}$. Dans les pays d'Europe continentale, le gaz est pour l'essentiel importé de l'extérieur, Russie, Algérie ou Norvège, dans le cadre de contrats à long terme, indexés sur le prix du pétrole.

Sur le marché britannique, les transactions entre producteurs et fournisseurs opèrent de façon transparente sur un nombre limité de «places de marché » dont la principale est le « National Balancing Point ». Grâce à cette place de marché, les prix du gaz sur le marché de gros sont connus. Ils prennent la forme de prix spot (au jour le jour) ou de prix à terme (à 3 mois, 6 mois, 1 an...). Un prix de vente proposé par un fournisseur à ses clients industriels pour un contrat de fourniture du gaz s'appuie sur cette référence : le prix de vente au client est défini par une formule indexée, l'indice étant calculé sur la base des prix affichés sur la place de marché (le NBP). La formule de prix pour le client est généralement le reflet de l'approvisionnement du fournisseur.

\footnotetext{
${ }^{2}$ Il s'agit de plateforme électronique qui fonctionnent sur le principe des bourses, rassemblent l'ensemble des transactions et affichent les prix et volumes échangés.
} 
Ainsi, les prix de contrats échangés sur la place de marché constituent la référence pour un ensemble important de transactions. $\mathrm{Y}$ compris pour les activités financières, qui se développent parallèlement. Ainsi, sur le marché britannique, l'existence d'une place de marché transparente a permis aux banques d'investissement de développer des services financiers indépendamment des producteurs et fournisseurs. Les clients industriels font appel directement à des banques spécialisées pour gérer le risque associé à ces formules fluctuantes.

Les pays européens «continentaux » n'ont pas de production sur leur sol et sont dépendants d'importations en provenance des pays producteurs : avant la mise en place de la politique de libéralisation, ces importations étaient organisées sur la base de contrats à long terme indexés sur les produits pétroliers. Ces contrats à long terme étaient considérés comme le meilleur moyen de financer les lourds investissements d'exploitation et de transport (Boussena, S. et al. 2006). La libéralisation des marchés n'a pas fondamentalement changé cette pratique en France, même si la Commission européenne souhaite sa disparition pour accélérer l'ouverture du marché à la concurrence. En 2003, selon la Commission de Régulation de l'Energie, environ 95\% du gaz importé en France était acheté dans le cadre de contrats à long terme (10 à 20 ans) aux producteurs de gaz. Lors de l'enquête, les places de marchés (le Zeebrugge en Belgique et le TTF aux Pays-Bas) ne représentent qu'une part très faible des approvisionnements totaux.

Contrairement à la Grande-Bretagne, l'importance des contrats à long terme dans l'achat de gaz en Europe continentale n'a pas permis l'établissement d'une référence de marché propre au gaz. Comme il n'existe pas de référence partagée entre fournisseurs et avec les clients, les fournisseurs sont relativement libres dans la fabrication de leur prix. Les fournisseurs combinent leur portefeuille d'approvisionnement avec des produits financiers afin d'offrir à leur client une diversité de structures de prix, des prix fixes et des prix indexés. Les formules d'indexation peuvent utiliser des indices à long terme des produits pétroliers (comme le 
«Brent») ou des indices à court terme des marchés de gros du gaz (comme le «Zeebrugge »). Les fournisseurs s'appuient sur des couvertures financières qui prennent en charge le risque financier d'un écart entre la formule de prix à laquelle ils achètent leur gaz (leur portefeuille d'approvisionnement, pour l'essentiel les contrats à long terme) et la formule (ou le prix fixe) proposée au client.

Autrement dit, le prix indexé est une construction sophistiquée qui tente de pallier l'absence de référence de prix du gaz continental partagée par tous les acteurs, par un recours à des références de prix issues d'un autre marché, celui des produits pétroliers... Cette construction s'appuie sur la base d'indices pétroliers qui agissent comme des «prothèses » (Caliskan, 2007) en l'absence de référence gazière partagée. Cet échafaudage complexe de références et d'instruments laissent la place à une diversité de représentations, d'interprétations et de pratiques. Suivant l'indice choisi (gaz ou pétrole), un acheteur de gaz aura deux lectures tout à fait différentes des variations de prix et de la compétitivité des offres.

\section{SIMPLIFICATION DES REPRESENTATIONS DU MARCHE PAR LES}

\section{CONSULTANTS ET FOURNISSEURS}

Les premières années de la libéralisation des marchés du gaz en France et en Allemagne, la définition des pratiques et des formes de transactions sur ces marchés fait l'objet d'une compétition entre deux types d'acteurs : les fournisseurs en place, (ex-monopoles comme Gaz de France, E.On, Distrigaz...), et les fournisseurs présents en Grande-Bretagne, qui ont déjà connu une libéralisation quelques années plus tôt, et qui ont développé des activités de conseil en Europe continentale. Ces deux types fournisseurs ne communiquent pas les mêmes représentations du marché, ils ne défendent pas les mêmes pratiques d'achat. Par des 
représentations simplifiées du marché, ils tentent chacun de leur côté d'orienter les acheteurs vers les pratiques qui valorisent le mieux leurs ressources.

Commençons par les consultants, issus généralement des fournisseurs de Grande-Bretagne ou des pays scandinaves. N'ayant pas les réserves de gaz ou les capacités de transports suffisantes pour entrer rapidement sur les nouveaux marchés continentaux, ces fournisseurs ont développé une activité de conseil pour s’imposer comme prescripteurs du marché, influencer les pratiques d'achat et accélérer l'ouverture. Ils parient sur le développement des places de marché existantes en Europe continentale et ils considèrent que ces places de marché deviendront la référence de prix. Ils communiquent à leur client sur le modèle du marché britannique, où l'indice NBP constitue la référence pour la majeure partie des transactions. Ils conseillent à leur client de s'appuyer sur des formules indexées sur les prix définis sur la place de marché de Zeebrugge. Le choix de cette référence de prix correspond aussi au fait que ces acteurs interviennent principalement sur le NBP et souhaitent promouvoir les prix spot comme une référence de prix pour le reste de l'Europe. De même, ils transposent sur les marchés continentaux les pratiques d'achat développées sur les marchés déjà libéralisés : l'organisation d'un appel d'offre à une date donnée, prévue à l'avance, pour acheter un certain volume de gaz, sur la base d'une formule de prix indexée. Cette méthode permet au consultant de développer son rôle : il aide l'acheteur à comparer les formules proposées par les fournisseurs par une simulation sur la base des évolutions probables des indices.

A côté des consultants, les principaux fournisseurs «continentaux » ont développé leur expertise et se sont associés à des banques partenaires pour fournir une «offre intégrée » combinant gaz physique et couverture financière adaptée. Leur stratégie est la suivante : accompagner au mieux la transition des pratiques d'acheteurs industriels, éviter que les consultants ne s'interposent, tout en faisant la démonstration que les anciens monopoleurs 
publics se sont réellement convertis au marché. Les fournisseurs ne communiquent pas la même réalité du marché que les consultants. Ils expliquent qu'il est préférable de s'appuyer sur les indices pétroliers, puisqu'ils se fournissent eux-mêmes avec des contrats indexés avec ces indices. Ils alimentent les clients en informations sur les marchés pour les convaincre que les prix sur les marchés spot existants (Zeebrugge, NBP) ne sont pas représentatifs des portefeuilles d'approvisionnement des fournisseurs. Les banques partenaires, spécialisées en trading de commodités, défendent aussi l'indexation du gaz sur les produits pétroliers : elle leur permet d'offrir les mêmes services d'anticipation des prix et de couverture financière que ceux qu'ils offrent déjà aux acteurs du marché pétrolier.

Enfin, les fournisseurs déconseillent l'appel d'offre annuel préconisé par les consultants et défendent l'achat «au bon moment », une pratique qui s'appuie sur une consultation régulière des fournisseurs : l'acheteur définit avec un ou plusieurs fournisseurs son besoin, le type de formule de prix. Ces fournisseurs l'informent régulièrement sur leurs prix, en particulier quand ceux-ci sont attractifs. Et l'acheteur contracte avec eux quand il pense pouvoir faire une bonne affaire.

Ainsi, il existe une certaine rivalité entre acteurs du champ dans la définition des bonnes pratiques d'achat de gaz. Les acteurs en quête d'une position dominante rivalisent dans la communication de représentation du fonctionnement du marché, des risques, des bonnes pratiques d'achat. Ainsi, on constate que les consultants pratiquent un sensemaking offensif : ils présentent aux clients industriels une vision simplifiée du marché, une vision idéalisée cohérente avec la libéralisation, mais en décalage avec une réalité caractérisée par le maintien des contrats à long terme. Inversement, les fournisseurs en place pratiquent un sensemaking prudent : ils défendent les pratiques les plus conformes à la réalité du marché. Ils encouragent une prise en compte des indices de prix mais freinent l'exercice de la mise en concurrence par le client. 
Tableau 1. Représentations simplifiées du marché : « marché gazier » versus « marchés pétroliers »

\begin{tabular}{|c|c|c|}
\hline & $\begin{array}{c}\text { Représentation et pratiques } \\
\text { privilégiées } \\
\text { par les consultants }\end{array}$ & $\begin{array}{c}\text { Représentation et pratiques } \\
\text { privilégiées } \\
\text { par les fournisseurs } \\
\text { « continentaux » }\end{array}$ \\
\hline Marché & $\begin{array}{l}\text { Le prix du gaz se forme sur une place } \\
\text { de marché où producteurs et } \\
\text { fournisseurs échangent entre eux }\end{array}$ & $\begin{array}{l}\text { Le prix du gaz résulte des contrats } \\
\text { d'approvisionnement à long terme du } \\
\text { gaz, indexés sur le prix du pétrole }\end{array}$ \\
\hline Choix des indices & $\begin{array}{l}\text { Les indices «gaziers » sont } \\
\text { représentatifs du marché }\end{array}$ & $\begin{array}{l}\text { L'indexation pétrole est représentative } \\
\text { de la majorité des échanges }\end{array}$ \\
\hline Stratégie d'achat & $\begin{array}{l}\text { L'absence de transparence sur le prix } \\
\text { justifie la mise en concurrence } \\
\text { systématique par appel d'offre }\end{array}$ & $\begin{array}{l}\text { La mise en concurrence par appel } \\
\text { d'offre empêche l'achat «au bon } \\
\text { moment» }\end{array}$ \\
\hline Gestion des risques & $\begin{array}{l}\text { Gestion du risque de variation de prix } \\
\text { avec de banques spécialisées, avec } \\
\text { des produits dérivés du marché gazier }\end{array}$ & $\begin{array}{l}\text { Gestion du risque de prix intégré dans } \\
\text { le contrat avec le fournisseur, sur la } \\
\text { base de l'indexation pétrole et de } \\
\text { produits dérivés du marché pétrolier }\end{array}$ \\
\hline $\begin{array}{l}\text { Détection des } \\
\text { opportunités de } \\
\text { marchés, suivi des } \\
\text { variations de prix }\end{array}$ & $\begin{array}{l}\text { Le marché gazier } \text { offre des } \\
\text { opportunités de prix bas }\end{array}$ & $\begin{array}{l}\text { Le marché pétrolier est très « liquide» } \\
\text { et offre des prix moins variables et plus } \\
\text { prévisibles }\end{array}$ \\
\hline
\end{tabular}

\section{LE SENSEMAKING A L'GUVRE : DONNER SENS AUX INCERTITUDES}

Les acheteurs sont donc confrontés à deux discours, contradictoires entre eux, sur le marché et sur les pratiques d'achat « efficaces ». Dans un premier temps, une large majorité des clients industriels que nous avons interrogés ont adhéré au discours véhiculé par les consultants, en particulier jusqu'en 2005. A partir de 2005, leur attitude évolue. Ils sont de plus en plus sensibles au discours des fournisseurs historiques qui retrouvent une forte crédibilité. Une majorité d'entre eux abandonnent les appels d'offre à date fixe, puis abandonnent les indexations des marchés spot du gaz, Zeebrugge ou NBP.

Nous expliquons l'adoption des pratiques préconisées par les consultants, puis leur abandon au profit des pratiques défendues par les fournisseurs, comme le résultat d'une activité de 
construction de sens par les acheteurs: cette activité s'appuie sur des schémas d'interprétations hérités ou développés à cette occasion, et sur des signaux générés par le marché : la variation des prix du gaz et des indices pétroliers.

\subsection{MISE EN CONCURRENCE SYSTEMATIQUE VERSUS DECISION D'ACHAT REACTIVE}

L'adhésion d'une partie des grands consommateurs industriels français aux discours des consultants s'explique par la cohérence de celui-ci avec l'annonce de l'ouverture des marchés : le choix des indices gaziers était assez raisonnable à partir du moment où l'on s'attendait à une baisse des prix sur le marché de gros grâce à la libéralisation. L'organisation systématique des appels d'offre avait tout son sens dans un contexte où le prix de marché n'existait pas et où les pratiques d'approvisionnement des fournisseurs, en amont, restaient opaques. Elle s'inscrivait totalement dans la logique professionnelle que défendaient les fonctions achats. Les consultants avaient pour eux l'expérience de la libéralisation dans leur pays, ils se plaçaient comme des tierces parties indépendantes. Bref, le sensemaking offensif des consultants était cohérent avec les représentations partagées des changements en cours, c'est pourquoi bon nombre d'acheteurs adhèrent.

A priori, les fournisseurs continentaux étaient dans une position moins favorable. Du point de vue des clients, dans le contexte d'ouverture du marché, les fournisseurs «historiques» avaient moins de légitimité que les consultants à les aider à définir leur stratégie d'achat. Ils étaient considérés comme juges et parties, attachés à leur position monopolistique. Néanmoins, ils sont parvenus à reconstruire leur légitimité, par une très forte présence auprès des clients industriels. Ils avaient avec eux la forte inertie de l'organisation du marché, qui n'a pas évolué aussi vite que les consultants le concevaient. C'est ainsi qu'ils sont parvenus à défendre l'indexation pétrole et qu'ils ont disqualifié l'indexation sur le prix spot ou future du marché gazier, soumis dans cette période à de très fortes variations. 
Ayant regagné une légitimité sur l'utilisation des indices pétroliers comme référence pour les contrats avec les clients, les commerciaux des fournisseurs ont pratiqué un sensemaking offensif sur la gestion des risques prix associés à ces indices. Les clients étaient invités à des conférences où les analystes présentent les évolutions récentes du marché et leurs techniques pour les appréhender.

Après l'expérience de plusieurs appels d'offres, la totalité des acheteurs interrogés adhéraient à l'idée communiquée par les commerciaux des fournisseurs, que : "Ce qui fait le prix, c'est le marché. Le moment de l'achat est beaucoup plus important que la négociation de la marge $d u$ fournisseur ». Sous l'influence des commerciaux, les acheteurs ont déplacé leurs investissements vers le suivi des indices pétroliers. Ils ont trouvé dans l'anticipation des évolutions de marché et dans l'intervention sur ces marchés une opportunité pour développer une fonction achat spécialisée et valorisée, et se sont concentrés sur cet aspect de leur activité. Beaucoup d'acheteurs ont abandonné les appels d'offre à date fixe : ils ont pris conscience que ces procédures affaiblissaient leur réactivité.

L'influence des commerciaux et des analystes sur les acheteurs s'est construite par un accompagnement quasiment quotidien. Ils parviennent ainsi à démontrer leur compétence par une accumulation d'informations, d'interprétations, de conseils d'achat qui contribuent progressivement à la stabilisation de repères par l'acheteur. L'information par les commerciaux et sa réinterprétation par les acheteurs ont créé une relation singulière marquée par la dépendance de l'acheteur vis-à-vis de son conseiller, similaire à la relation entre gérants de fonds d'investissement et brokers (Ortiz, 2005).

Ce sensemaking des fournisseurs s'appuyait aussi sur la disqualification des représentations alternatives. Ainsi, les commerciaux des fournisseurs ont démontré que la place de marché Zeebrugge était essentiellement un marché d'ajustement entre fournisseurs, qu'elle ne 
concernait qu'une frange limitée des échanges et qu'elle n'était pas représentative des coûts d'approvisionnement. Par exemple, lors de la hausse des prix spots du gaz sur le marché du NBP et de Zeebrugge, les commerciaux des fournisseurs se sont empressés de stigmatiser un prix «dépendant des conditions climatiques et des aléas techniques » et donc, présentant de fortes incertitudes.

Ce sensemaking des fournisseurs a produit le «cadre »(Beunza, Garud, 2007) dans lequel les acheteurs raisonnent. Les acheteurs se sont représenté le «marché du gaz » comme quasiment « intégré » au marché des produits pétroliers et ne s’intéressaient plus au marché spot du gaz. Leur représentation était confirmée par le fait que les formules d'indexation pétrole proposées par les différents fournisseurs se ressemblaient.

Dans la totalité des relations étudiées dans l'enquête, les commerciaux du fournisseur parviennent, grâce à une relation approfondie, quotidienne, avec leur client, à stabiliser une représentation du marché et une pratique d'achat, à la fois la plus proche de l'expérience de l'acheteur et qui valorise au mieux les ressources du fournisseur.

Cependant, dans un contexte de marché incertain, les commerciaux courent un risque : la brutale prise de conscience par le client de la fragilité des représentations sur lesquelles il se fonde. Bref, la robustesse de la relation commerciale dépend aussi de sa capacité à communiquer sur un marché incertain et complexe, autrement dit, à pratiquer un sensemaking prudent et à encourager son client à faire de même. Par exemple, lorsque les prix spot et future du marché du gaz à Zeebrugge se sont effondrés en 2006 et 2007, les commerciaux ont eu quelques inquiétudes. Les acheteurs, qui considéraient jusqu'à présent que l'indexation pétrole était la seule référence, pouvaient devenir très suspicieux. Cette situation faisait courir le risque que les acheteurs accèdent par les concurrents ou les consultants à une autre représentation du marché. Un travail commercial important a été entrepris pour préciser la 
nature des indices, leur représentativité, les raisons de sa baisse, les risques associés aux prix spot...

\subsection{L'ALLONGEMENT DE L'HORIZON TEMPOREL DES ANTICIPATIONS}

L'anticipation des évolutions des prix est une activité sujette aux tentations d'un « sensemaking offensif ». Au fur et à mesure de notre enquête, nous avons observé que les commerciaux adoptaient un discours de plus en plus prudent avec leur client à propos des évolutions probables des prix. Les responsables des ventes (obéissant aux recommandations des autorités des marchés financiers) ont recadré les pratiques commerciales pour qu'elles se limitent à informer les clients sur les prix et qu'elles abandonnent les conseils d'achat.

Néanmoins, toutes les interactions entre commerciaux et clients se passent par téléphone et ces échanges téléphoniques sont le lieu d'un jeu ambigu : les commerciaux répondent aux attentes de l'acheteur en termes d'anticipation de tendance et de conseil, sans pour autant s'engager. Le commercial donne ses conseils de façon implicite, en privilégiant une information parmi l'ensemble des informations communiquées : par exemple, un événement, une tendance, qui peut donner à penser qu'il est préférable d'attendre ou non pour fixer un prix. En jouant sur l'ambiguïté, le commercial doit faire accepter au client qu'il est seul responsable de la décision.

Interrogés sur cette question, les acheteurs admettent être conscients qu'au-delà de l'information, la façon dont leur conseiller présente cette information les conduit à favoriser une décision plutôt qu'une autre. Les acheteurs assument l'ambiguïté de l'interaction et se saisissent de ce qu'ils considèrent comme des conseils d'achat.

Se dégagent clairement plusieurs profils d'acheteurs, selon leur capacité à gérer cette tension propre à l'action dans un contexte incertain. Certains acheteurs ne perçoivent pas de façon 
précise les contradictions et les incertitudes qui pèsent sur leur activité. Ils réinterprètent chaque nouvelle information sans remettre en question leur schéma d'interprétation et leur pratique. Parmi eux, des acheteurs (en général, quand il s'agit de dirigeants qui assurent l'achat d'énergie en plus de leurs autres fonctions) réinterprètent les anticipations dans leur culture budgétaire, et «allongent» 1'horizon temporel des anticipations. L'ambigüité des discours et des anticipations communiquées par les analyses offre suffisamment d'espace de jeu pour que les acheteurs reconstruisent leur propre cohérence : ils survalorisent les capacités d'anticipation des analystes. Quand le marché suit une évolution inattendue, ils renvoient la responsabilité aux commerciaux de les avoir insuffisamment alertés. On peut considérer qu'ils pratiquent un sensemaking défensif.

D'autres acheteurs, à l'inverse, adhèrent totalement à la culture de trading: l'analyse rétrospective des évolutions du marché les encourage dans la croyance que la maîtrise de cette activité d'achat est possible à condition de travailler sur un court terme, d'accumuler de l'expérience et de s'appuyer sur les bons informateurs. Ils vivent les exigences de prévision budgétaire comme une source de paralysie. Les retournements inattendus les surprennent, mais ne remettent pas en question leur stratégie. Ils pratiquent un sensemaking offensif.

Enfin, d'autres acheteurs expriment de nombreux doutes sur les représentations, les interprétations, les conseils que les commerciaux communiquent. Leur investissement dans le suivi des marchés les conduit à prendre conscience de la faiblesse des prévisions : "On se rend compte que les fondamentaux qui pouvaient exister, comme les faits saisonniers, n'existent plus. Il y a des analyses intéressantes, mais ils (les analystes) sont dans le brouillard, ils sont comme nous ». Ce doute rejaillit alors sur l'ensemble des conseils qu'ils prennent avec distance. Ils n'hésitent pas à exprimer l'inquiétude que leur inspirent les incertitudes du marché. Ils ne cherchent pas à habiller ou simplifier cette réalité, et se gardent d'adhérer à chaque « nouvelle illusion ». Ils pratiquent un sensemaking prudent. 


\subsection{ARTICULER LES INCERTITUDES DU MARCHE AVEC LA PREVISION BUDGETAIRE}

La pratique des couvertures des risques par des instruments financiers peut devenir une activité particulièrement sophistiquée. Les formules indexées des contrats gaziers sont compatibles avec toute une palette de produits financiers issus des marchés pétroliers (options, swaps...) qui permettent à tout moment de passer d'un prix variable à un prix fixe pour une partie des volumes de gaz sur une période donnée.

Pour construire sa stratégie, l'acheteur raisonne généralement par rapport à la culture budgétaire de son entreprise : selon que celle-ci autorise un prix d'achat incertain, ou limité dans une certaine fourchette, ou un prix fixe, pour telle ou telle échéance, trimestrielle ou annuelle. L'acheteur raisonne aussi à partir de sa compréhension des variations de prix sur le marché, des possibilités d'anticiper...

Beaucoup d'entreprises disposent d'une pratique budgétaire à horizon annuel et demande à leur acheteur de fixer le prix d'achat du gaz une année sur l'autre. L'acheteur qui a pris conscience des variations de prix comprend que fixer un prix pour une année, c'est prendre un risque important d'être «à côté du marché » en particulier si celui-ci baisse. Décider de fixer un prix suppose d'anticiper un prix de marché un an à l'avance! Une phrase revient régulièrement dans les entretiens : «Pour ceux qui connaissent les marchés, fixer un prix pour l'année à venir, c'est spéculer. Pour les autres, conserver un prix fluctuant, c'est spéculer».

Les fournisseurs ont intégré cette ambivalence des entreprises clientes et ils ont proposé des stratégies de plus en plus sophistiquées de lissage du risque. Selon eux, la possibilité de fixer le prix progressivement, trimestre par trimestre, en fractionnant la quantité à fixer, est le meilleur moyen de ne pas prendre une position risquée. Cette stratégie de fractionnement a rencontré un succès indéniable auprès des acheteurs parce qu'elle articulait astucieusement 
culture de marché avec la culture industrielle de budget fixé en partie à l'avance. Le succès de cette stratégie était d'autant plus important qu'elle s'est développée dans un contexte de marché du pétrole haussier : fixer un prix pour les 12 mois à venir était presque toujours payant. Néanmoins cette stratégie est devenue un piège redoutable lors de la chute du prix du pétrole en septembre 2008 : malgré la chute du prix du pétrole, les industriels ont continué à s'approvisionner en gaz pendant plusieurs mois à des prix négociés avant la chute.

Cette stratégie de couverture s'appuyait sur une hypothèse implicite d'un marché haussier d'une année sur l'autre. L'importance de cette hypothèse n'était jamais vraiment formulée ni par les analystes, ni par les clients, tellement elle était tenue pour évidente. La sophistication des pratiques a masqué la fragilité des hypothèses sur lesquelles elles s’appuyaient.

Plus globalement, les commerciaux sont tentés d'asseoir leur légitimité en défendant des stratégies de plus en plus sophistiquées et valorisées d'anticipation des évolutions des prix et de gestion des risques et de ne pas assumer la part incertaine et la fragilité des fondements à partir desquels ils raisonnent. S'ils communiquent excessivement leurs hésitations, ils risquent de ne pas convaincre les clients d'adopter leurs offres. Réciproquement, s'ils ne communiquent pas les hésitations, ils risquent de perdre le crédit de leurs clients lors d'événements inattendus qui contredisent leurs raisonnements.

Tableau 2 : Sensemaking offensif, prudent et défensif pour chaque incertitude du marché gazier 


\begin{tabular}{|c|c|c|c|}
\hline & Sensemaking offensif & $\begin{array}{c}\text { Sensemaking } \\
\text { prudent }\end{array}$ & $\begin{array}{c}\text { Sensemaking } \\
\text { défensif }\end{array}$ \\
\hline $\begin{array}{l}\text { Représentations } \\
\text { du marché }\end{array}$ & $\begin{array}{l}\text { Une représentation } \\
\text { univoque du marché : la } \\
\text { bourse de gaz Zeebrugge } \\
\text { est considérée comme } \\
\text { représentant le marché de } \\
\text { gros du gaz dans son } \\
\text { ensemble }\end{array}$ & $\begin{array}{l}\text { Acceptation d'une } \\
\text { pluralité de forme de } \\
\text { transaction sur le marché } \\
\text { de gros, contrats à long } \\
\text { terme indexé pétrole et } \\
\text { place de marché de vente } \\
\text { de gaz. }\end{array}$ & $\begin{array}{l}\text { Une représentation } \\
\text { univoque du marché : le } \\
\text { gaz est assimilé à un } \\
\text { produit pétrolier comme le } \\
\text { fioul, l'essence... }\end{array}$ \\
\hline $\begin{array}{l}\text { Pratique d'achat et } \\
\text { de suivi des prix }\end{array}$ & $\begin{array}{l}\text { Pratique systématique de } \\
\text { l'appel d'offre ou stratégie } \\
\text { d' «achat au bon } \\
\text { moment » } \\
\text { Suivi d'une seule } \\
\text { référence de prix } \\
\text { (Zeebrugge) }\end{array}$ & $\begin{array}{l}\text { Pratiques de } \\
\text { consultations régulières, } \\
\text { parfois de l'appel d'offre. } \\
\text { Suivi de l'ensemble des } \\
\text { références de prix, souci } \\
\text { de comprendre la part de } \\
\text { chacune, son évolution }\end{array}$ & $\begin{array}{l}\text { Stratégie d' « achat au bon } \\
\text { moment » } \\
\text { Suivi d'une seule } \\
\text { référence de prix (Pétrole) }\end{array}$ \\
\hline $\begin{array}{l}\text { Sophistication } \\
\text { des pratiques de } \\
\text { couverture des } \\
\text { risques } \\
\end{array}$ & $\begin{array}{l}\text { Forte sophistication des } \\
\text { stratégies de couverture }\end{array}$ & $\begin{array}{l}\text { Faible sophistication, } \\
\text { usage contingent du prix } \\
\text { variable ou fixe }\end{array}$ & $\begin{array}{l}\text { Faible sophistication, } \\
\text { utilisation du prix fixe }\end{array}$ \\
\hline $\begin{array}{l}\text { Horizon temporel } \\
\text { des anticipations }\end{array}$ & $\begin{array}{l}\text { S'oppose autant que } \\
\text { possible à la logique } \\
\text { budgétaire et cherche à } \\
\text { s'adapter à la réactivité } \\
\text { nécessaire pour tirer } \\
\text { bénéfice du marché }\end{array}$ & $\begin{array}{l}\text { Essaie d'adapter sa } \\
\text { stratégie en fonction d'un } \\
\text { horizon raisonnable de } \\
\text { prévision }\end{array}$ & $\begin{array}{l}\text { Allongement des } \\
\text { anticipations proposées } \\
\text { par les analystes pour } \\
\text { «coller» avec la logique } \\
\text { budgétaire, }\end{array}$ \\
\hline $\begin{array}{c}\text { Contribution à } \\
\text { l'institutionnalisation }\end{array}$ & $\begin{array}{l}\text { Forte institutionnalisation } \\
\text { des nouvelles pratiques et } \\
\text { techniques, diffusion } \\
\text { rapide, mais qui reste } \\
\text { assez peu robuste face aux } \\
\text { retournements de marché }\end{array}$ & $\begin{array}{l}\text { Faible, diffusion lente, } \\
\text { mais plus robuste face } \\
\text { aux retournements de } \\
\text { marché }\end{array}$ & $\begin{array}{l}\text { Absence } \\
\text { d'institutionnalisation de } \\
\text { nouvelles pratiques. }\end{array}$ \\
\hline
\end{tabular}

\section{Discussion : COMment le tRAVAIl DE CONSTRUCTION DE SENS CONTRIBUE A}

\section{L'INSTITUTIONNALISATION DE SCHEMAS COGNITIFS}

Notre étude de cas contribue à la compréhension des dynamiques d'institutionnalisation dans un champ en émergence. Les pratiques que nous avons observées ne sont pas complètement institutionnalisées, autrement dit, intériorisées par l'ensemble des acteurs du champ. En effet, on ne trouve pas d'institutions stabilisées, au sens de Scott (2003), c'est-à-dire des pratiques efficaces définitivement tenues pour évidentes. Il existe une diversité de pratiques, plus ou moins cohérentes avec des schémas interprétatifs partagés, définissant des moyens d'action ou des buts légitimes. La libéralisation des marchés génère une compétition entre pratiques 
incomplètement institutionnalisées : des pratiques héritées du passé et remises en causes - les contrats long terme, l'indexation pétrole - des pratiques empruntées à d'autres marchés, qui sont souvent idéalisées - la place de marché «transparente » et « liquide ».

Face à une situation d'incohérence et d'incertitude institutionnelle, les acteurs réinvestissent dans l'interprétation dans la construction de sens (Weber, Glynn, 2006). Selon Weick (1993, 2005), cette activité de construction de sens peut prendre plusieurs formes. Dans le cours ordinaire de l'action, des pratiques routinières et des schémas d'interprétation intériorisés s'imposent à l'acteur. Le travail de construction de sens résout naturellement les dissonances cognitives et reconstruit une réalité perçue cohérente avec nos schémas préétablis. Dans certaines situations critiques, l'expérience concrète, les exigences immédiates de la situation d'action peuvent conduire à un renouvellement dans la construction de sens. C'est aussi le cas lors de la prise de conscience de fortes contradictions entre «institutions » (Weber, Glynn, 2006). L'accumulation de signaux contradictoires produit un inconfort tel que l'individu accepte d'abandonner certains schémas préconçus. Le processus de construction de sens reprend son autonomie et fait des choix entre les schémas institutionnalisés disponibles (Weber, Glynn, 2006). En général la remise en question est rétrospective (Weick, 2006) : il faut être allé jusqu'au bout de son action, faire l'expérience des contradictions pour accepter de sortir de ses schémas préconçus.

Nos observations confirment cette dualité dans les activités de construction de sens. La recherche de cohérence explique pourquoi les acheteurs de gaz, dans les débuts de l'ouverture des marchés, ont adhéré au discours des consultants, lesquels s'appuyaient sur un certain nombre de stéréotypes comme la mise en concurrence systématique, le prix spot comme référence... Cette adhésion était d'autant plus forte qu'elle était cohérente avec leur nouveau rôle. Elle explique aussi la sophistication des pratiques de suivi des indices et de couverture de risque, la grande confiance dans l'interprétation des variations des prix et dans les 
anticipations. Toutes ces pratiques relèvent d'un sensemaking offensif fondé sur une adhésion univoque aux nouvelles représentations et nouvelles méthodes sophistiquées. Ce sensemaking offensif s'est matérialisé dans des outils et des règles formalisées (Weick, 1995). Les acheteurs ont été parfois prisonniers des procédures sophistiquées et des cadres d'interprétation qu'ils ont contribué à mettre en place. Voilà pourquoi une partie des acheteurs ont tardé à réviser leur représentation du marché et leur stratégie.

Néanmoins, nous avons aussi observé des situations de réélaboration du sens, facilitée par l'existence d'un répertoire de représentations et de pratiques institutionnalisées (Weber, Glynn, 2006). A chaque situation critique, les acheteurs puisent dans des ressources cognitives alternatives pour forger leur compréhension et leur action: par exemple, la légitimité des contrats gaziers à long terme et de l'indexation pétrole s'appuie sur un important historique, les pratiques d'achat et de couverture des marchés pétroliers sont facilement transposées à l'achat de gaz... Ils reconstruisent leur interprétation d'autant plus facilement que d'autres cadres interprétatifs leur ont été communiqués et ont été considérés comme acceptables, cohérents...C'est aussi le cas des acheteurs qui comprennent et acceptent que les horizons d'anticipation des prix par les analystes ne correspondent pas aux horizons d'anticipation budgétaires. Ils acceptent davantage le maintien d'une contradiction organisationnelle, et de la dissonance cognitive qu'elle produit. Ils pratiquent un « sensemaking prudent ».

Nous avons constaté combien le commercial pouvait contribuer au travail de construction de sens par l'acheteur. Il peut être utile de distinguer la construction de sens par le commercial qui est communiquée à l'acheteur et l'activité de construction de sens par l'acheteur. Le commercial est en mesure d'influencer le processus de construction de sens vers une redéfinition préférée de la réalité, ce que Gioia et Chittipeddi (1991) nomment le «sensegiving ». Cette influence peut s'appuyer sur des discours mais elle opère aussi dans le 
cadre d'interactions plus routinières, dans l'action, permettant une réélaboration du sens davantage appropriée et partagée (Balogun, Johnson, 2005). La présence du commercial, au cœur de l'expérience de l'acheteur, réintroduit de la cohérence, dissimule les faiblesses du marché comme l'absence de liquidité et la «prothèse »(Caliskan, 2007) que constitue la formule d'indexation pétrole qui permet d'assimiler le gaz à un produit pétrolier. Le commercial fournit les simplifications et les interprétations permettant la constitution d'une capacité d'action de l'acheteur. De même l'acheteur intervient-il de la même façon auprès de ses dirigeants.

Ainsi, le sensemaking est l'activité par laquelle des ressources cognitives faiblement institutionnalisées se consolident progressivement. Le sensegiving facilite l'activité de sensemaking. Sensemaking et sensegiving font partie des activités que Lawrence et Suddaby (2006) désignent par «travail d'institutionnalisation». Autrement dit, le travail d'institutionnalisation ne s'appuie pas seulement sur la production d'une argumentation convaincante et l'articulation aux schémas cognitifs existants, il s'agit de construction intersubjective de significations, au quotidien, en situation, face aux événements et de consolidation de celles-ci malgré la diversité des signaux contradictoires.

Nous avons montré que la construction de sens en situation de «crise » implique un renouvellement des schémas d'action et d'interprétation. Elle est d'autant plus accessible que l'on a accès à une pluralité de schémas en partie institutionnalisés. La situation de crise sera vécue de façon d'autant plus critique que l'on ne dispose pas de ressource pour l'interpréter. Or nous avons montré que le sensegiving tient un rôle essentiel dans la mise à disposition des différentes ressources cognitives dont nous disposons. Autrement dit, dans la situation étudiée, le fait que le commercial communique à l'acheteur une pluralité de schémas d'action et d'interprétation accroit les chances de l'acheteur de réélaborer ses repères en situation de crise, voire de prévenir la situation de crise en l'anticipant davantage. Un sensegiving prudent 
maintient cette pluralité. Un sensegiving offensif, trop univoque présente un risque : maintenir durablement les acteurs du champ dans l'illusion d'une solidité des représentations et des pratiques, institutionnaliser excessivement des schémas finalement assez fragiles.

Ainsi, le sensemaking offensif accélère les processus d'institutionnalisation mais néglige les événements révélateurs de sa fragilité, au risque de prendre toute crédibilité lors d'un retournement important. A l'opposé, il existe un sensemaking défensif qui réinterprète les évolutions du marché dans un cadre cognitif inchangé. Sensemaking offensif et défensif se rejoignent dans la mesure où les acteurs pensent avoir une maîtrise satisfaisante des incertitudes et du marché, souvent après avoir opéré d'importantes simplifications. Il y aurait enfin un sensemaking prudent qui encourage une distance critique, est conscient de la fragilité des représentations et des stratégies, accepte une représentation plus complexe du contexte, communique plusieurs schémas d'interprétation, limite la sophistication des outils et des procédures, si ceux-ci s'appuient sur des hypothèses incertaines. Le sensemaking prudent étant généralement la perspective dominante après quelques années d'expérience.

Les mêmes catégories peuvent être transposées à l'activité de sensegiving par les commerciaux, consultants (ou même acheteurs, vis-à-vis de leurs dirigeants). Dans le cas du marché gazier, les consultants avaient engagé un sensegiving offensif, mettant en valeur un modèle idéal de marché, alors que les fournisseurs pratiquaient plutôt un sensegiving défensif, mettant en valeur les institutions existantes comme l'indexation pétrole. Les commerciaux des fournisseurs se sont progressivement convertis à un sensegiving offensif puis prudent sur certains thèmes comme l'achat au bon moment, le choix des indices, l'horizon temporel des prévisions. Du côté des banques partenaires, on constate plutôt un sensegiving offensif favorisant une sophistication des pratiques de couverture des risques. 


\section{CONCLUSION}

Cet article propose de rendre compte des incertitudes qui pèsent sur le marché gazier libéralisé et de la façon dont les acteurs de ce marché les prennent en charge. Le marché gazier apparait comme un champ en cours d'institutionnalisation où les représentations et pratiques peinent à se stabiliser. Les fondamentaux de ce marché restent incertains et insaisissables.

Un détour par l'expérience des acteurs en situation met en valeur l'important investissement des commerciaux, des consultants, dans la construction de représentation, la communication de nouvelles pratiques, l'accompagnement des décisions. Il montre le caractère stratégique de cette activité en décrivant les rivalités entre ces acteurs dans la définition des représentations « vraies » des pratiques « efficaces ».

Enfin, l'article s'interroge sur la difficulté d'institutionnaliser de nouvelles pratiques dans un environnement évolutif. Au fur et à mesure que les acheteurs consolident et sophistiquent leurs pratiques, ils sont confrontés à des événements qui les invalident. L'article propose de distinguer trois attitudes en matière de «sensemaking » : une attitude «défensive », fidèle aux schémas et valeurs antérieurs à la libéralisation du marché, une attitude «offensive », confiante dans les représentations partagées et encourageant une sophistication des pratiques, et une attitude «prudente », qui combat les illusions et accepte l'incertitude. La dernière attitude, si elle ne permet pas une institutionnalisation rapide, semble mieux résister aux événements inattendus.

\section{Références bibliographiques}


J. Balogun and G. Johnson, "From Intended Strategies to Unintended Outcomes: The Impact of Change Recipient Sensemaking”, Organization Studies, vol 26, n 11, p: 1573-1601, 2005.

D. Beunza, R. Garud, "Calculators, lemming or frame-makers? The intermediary role of securities analysts”, in M. Callon, Y. Millo \& F. Muniesa (ed.), Market Devices, Sociological Review, 2007.

S. Boussena, Pauwels, J.-P., Locatelli, C., Swartenbroekx, C., Le défi pétrolier : questions actuelles du pétrole et du gaz, Vuibert, Paris, 496 p., 2006.

K. Caliskan, "Price as a market device: cotton trading in Izmir Mercantile Exchange", in M. Callon, Y. Millo \& F. Muniesa (ed.), Market Devices, Sociological Review. 2007.

D. Gioia, K. Chittipeddi, «Sensemaking and Sensegiving in Strategic Change Initiation », Strategic Management Journal, Vol. 12 , nº 6, pp 433-448, 1991.

A. Langley, "Strategies For Theorizing From Process Data", Academy of Management Review, vol. 24, n 4, 691-710, 1999.

T.B. Lawrence, Suddaby R., "Institutions and Institutional Work", in Clegg Steward, The Sage Handbook of Organization Studies, 2nd Edition, SAGE, 2006.

S. Maguire, Hardy C. Lawrence T.B., "Institutional Entrepreneurship In Emerging Fields: Hiv/Aids Treatment Advocacy In Canada", Academy of Management Journal, vol. 47, $\mathrm{n}^{\circ}$ 5, 657-679. 2004.

O. Ortiz, «Evaluer, apprécier : les relations entre brokers et gérants de fonds d'investissement », Economie rurale, 286-287, p 57-70, 2005. 
R.W. Scott, "Institutional carriers: Reviewing modes of transporting ideas over time and space and considering their consequences'. Industrial and Corporate Change Vol 12, 879894., 2003.

K. Weber and M.A. Glynn, "Making Sense with Institutions: Context, Thought and Action in Karl Weick’s Theory”, Organization Studies, vol 27, n¹1 p: 1639-1660, 2006.

K. E. Weick, "The collapse of sensemaking in organizations: The Mann Gulch disaster". Administrative Science Quarterly, vol 38, p. 628-652, 1993.

K. E. Weick, Faith, Evidence, and Action: Better Guesses in an Unknowable World, Organisation Studies, 21(11), pp. 1723-1736, 2006

K. E. Weick Sutcliffe K. M., Obstfeld D., «Organizing and the Process of Sensemaking », Organization Science, Vol. 16, No. 4, pp. 409-421, 2005. 